\title{
Effectiveness of MPEG-7 Color Features in Clothing Retrieval
}

\author{
Arsy Febrina Dewi ${ }^{1}$, Fitri Arnia ${ }^{2}$, Rusdha Muharar ${ }^{3}$ \\ ${ }^{1}$ Graduate of Electrical and Computer Engineering, Universitas Syiah Kuala, Aceh, Indonesia \\ ${ }^{2,3}$ Master of Electrical and Computer Engineering, Universitas Syiah Kuala, Aceh, Indonesia \\ *Corresponding author, e-mail: arsyfebrinadewi@yahoo.co.id', f.arnia@unsyiah.ac.id², \\ r.muharar@unsyiah.ac.id ${ }^{3}$
}

\begin{abstract}
Clothing is a human used to cover the body. Clothing consist of dress, pants, skirts, and others. Clothing usually consists of various colors or a combination of several colors. Colors become one of the important reference used by humans in determining or looking for clothing according to their wishes. Color is one of the features that fit the human vision. Content Based Image Retrieval (CBIR) is a technique in Image Retrieval that give index to an image based on the characteristics contained in image such as color, shape, and texture. CBIR can make it easier to find something because it helps the grouping process on image based on its characteristic. In this case CBIR is used for the searching process of Muslim fashion based on the color features. The color used in this research is the color descriptor MPEG-7 which is Scalable Color Descriptor (SCD) and Dominant Color Descriptor (DCD). The SCD color feature displays the overall color proportion of the image, while the DCD displays the most dominant color in the image. For each image of Muslim women's clothing, the extraction process utilize SCD and DCD. This study used 150 images of Muslim women's clothing as a dataset consistingclass of red, blue, yellow, green and brown. Each class consists of 30 images. The similarity between the image features is measured using the eucludian distance. This study used human perception in viewing the color of clothing. The effectiveness is calculated for the color features of SCD and DCD adjusted to the human subjective similarity. Based on the simulation of effectiveness DCD result system gives higher value than SCD.
\end{abstract}

Keywords: $C B I R, D C D, S C D$, eucludian distance, effectiveness

\section{Introduction}

Color is one of the most commonly used features in the retrieval process. Many objects can be recognized based on the colors contained. Color is also one of the features that fits perfectly with human vision. Almost every human being has the ability to see colors very well. Humans can recognize or choose something based on the colors they like or want. It also happens in the selection of clothing that want to be bought or used by humans. For example, in choosing fashion, humans will choose the appropriate color before buying or wearing the clothing. Clothing generally consists of one color or a blend of colors. But the human eye usually sees the most common colors of an object.

Clothing is a human used to cover the body. Clothing consist of dress, pants, skirts, and others. Clothing usually consists of various colors or a combination of several colors. The growing age, type, variety, and color of fashion, especially for women is also growing. This is due to the interest and the needs of the people who are increasingly looking for clothing. The barrier is the difficulty of describing the desired clothing at the searching process in written form. Therefore, people used a search method based on the color features of a female fashion image.

Content Based Image Retrieval (CBIR) is the methods of searching and calling image data based on the content of an image such as color, texture, and shape [1]. This study used color descriptor for the searching process. Color is one of the important things to search for female fashion. There are several descriptor color in MPEG-7 such as Scalable Color Descriptor (SCD) and Dominant Color Descriptor (DCD) [2]. SCD shows the color histogram of the image which displays the entire color proportion of an image. While DCD displays the most dominant color contained in the image. Based on previous research, both color descriptors seem good to use. Just so to know the color descriptor is the most suitable for use on women's clothing 
objects according to the perception of vision of the human eye. The application of color descriptor SCD and DCD in CBIR fashion system is a new research that has never been done before. Use of SCD and DCD will be tailored to the perception of humans to look at clothing.This study consisted of 150 datasets divided into 5 color categories: red, blue, yellow, green, and brown. Each category consists of 30 images of Muslim fashion. The image used was the image of Muslim women's clothing with a white background.

This research involves 10 opinions of respondents. Each respondent was asked to fill 10 sequences of images that were considered to have the closest color similarity to the query. The results were then matched with the system simulation results based on the SCD and DCD color features and the value of the effectiveness [10], [13] from each feature. Based on the simulation of F-Measure DCD result system gives higher value than SCD. In addition, the results of human perception analysis also provide higher effectiveness value for DCD, in which for the red category is 0.407 , blue category is 0.435 , green category is 0.403 , yellow category is 0.394 , and brown category is 0.478 . As for SCD, blue category is 0.416 , red category is 0.389 , green category is 0.387 , yellow category is 0.378 , and chocolate category 0.432 . This result showed that in this study DCD color features more suitable to human subjective analysis in choosing clothing. This study has the following contributions discusses the application of SCD and DCD features as color feature extraction for female Muslim dress, show the value of recall, precision, and F-Measure DCD higher than SCD, demonstrates that the use of DCD color features displays better results than SCD for female Muslim fashion objects based on human perception with effectiveness value.

\section{Material and Methods}

The simulation material consists of 150 images of Muslim women's clothing consisting of 5 categories namely red, blue, green, yellow, and brown. The size of the fashion image normalizes to a size of $215 \times 362$ pixels. The image of Muslim clothing used also cuts on the legs and face, Some examples of female muslin clothing images in each category are shown in Figure 1.
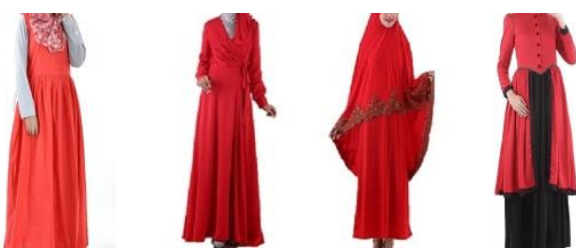

(a)
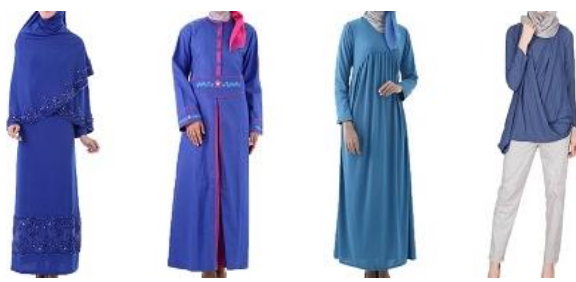

(b)
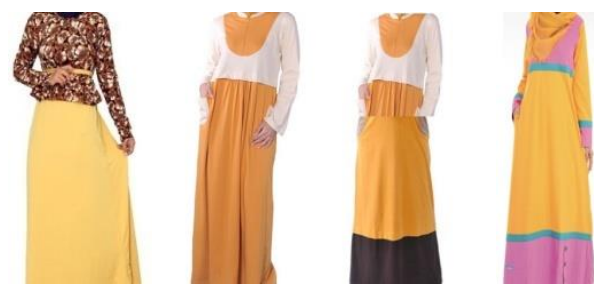

(c)

Figure 1. (a) Example of red category, (b) Example of blue category, (c) Example of yellow 
Figure 2. shows the process of CBIR system of female Moslem wear using SCD and DCD color features. The chart consists of the query side and the image dataset. In terms of query and dataset, the image of Muslim women's clothing undergoes the same process, which initially performed the pre-processing process such as changing the color space of the image from RGB to HSV to further perform which is the process of extracting SCD and DCD color features. After the feature was obtained, continue by matching process using eucludian distance. Furthermore, the results of SCD and DCD simulations were compared with the perception of 10 people in choosing clothing. The result of human perception was compared with the simulation result of the system and calculated the effectiveness value for each color feature.

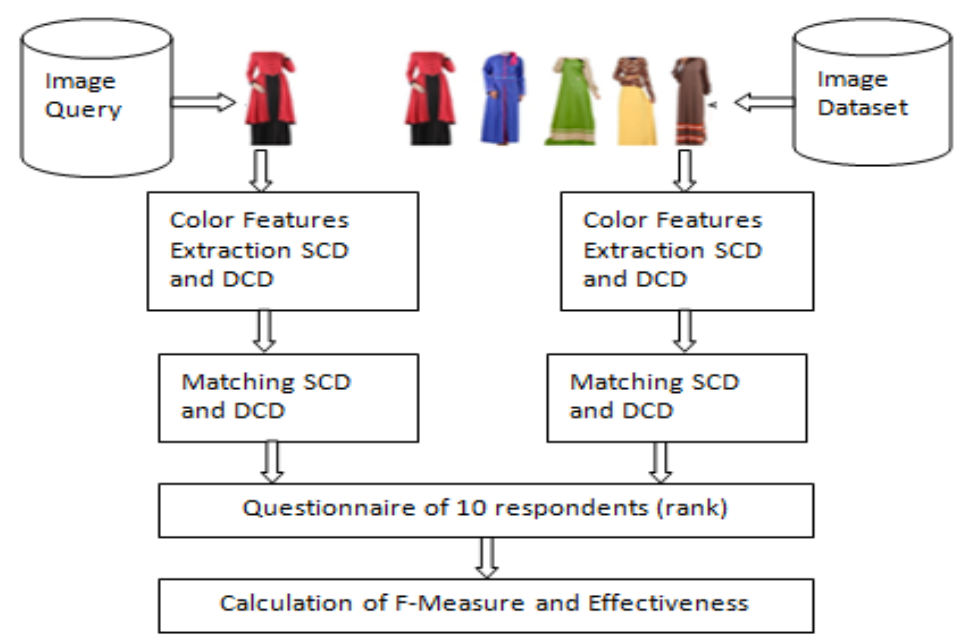

Figure 2. Block diagram using SCD and DCD color features

\subsection{Scalable Color Descriptor (SCD)}

SCD is one of the color descriptor in MPEG 7. SCD describes the color histogram of an image. SCD can be interpreted as Haar transformation applied to color histogram in HSV color space. The histogram color structure aims to identify the color distribution. SCD uses a uniformly quantized Hue Saturation Value (HSV) color space into 256-bin with 16 levels in H, and 4 levels respectively on $S$ and $V$. The histogram values are then represented/mapped into non-linear 4-bits. Haar transfomation is applied to histogram values that have been mapped into 4-bit [3].

The basic unit of Haar transformation consists of the addition and subtraction operation of one value with another value next to it. The basic unit of the Haar Transformation consists of high-pass and low-pass operations. This operation wasdone by summing and subtracting adjacent bin pairs. The high-pass coefficients can be discarded because the coefficients consist of information that was derived from the histogram [2].

\subsection{Dominant Color Descriptor (DCD)}

DCD is one of the color descriptor of MPEG-7. DCD describes the prominent color distribution in the image. This descriptor replaces the color information in the image with a small number of color representations. In other words, the number of colors generated by this descriptor is quite small, but still represents the overall color so that the image search process can be done quickly [5].

DCD can be denoted as follows:

$$
F=\left\{\left\{C_{i}, P_{i}\right\}, i=1,2, \ldots, N, P_{i} \in[0,1]\right\}
$$

where: $N=$ total number of dominant colors in the image

$C_{i}=$ the dominant color in sequence-i

$P_{i}=$ color percentage of $C_{i}$ 


\subsection{Image Similarity Size}

The similarity level between the two images in this study using Eucludian Distance. Eucludian Distance is a technique for calculating the distance between two vectors. Suppose two vector features are given $P=\{P 1, P 2, \ldots P n\}$ and $Q=\{Q 1, Q 2, \ldots Q n\}$, the distance between the two feature vectors $P$ and $Q$ is determined as follows:

$$
d=\sqrt{\sum_{i=1}^{n}(P n-Q n)^{2}}
$$

$P$ is the vector feature of the image $P$ and $Q$ is the vector feature of the $Q$ image, while $d$ is the distance between the vector features $P$ and $Q$.

\subsection{Valuation of CBIR System Performance}

To know the performance of CBIR system by doing the calculation process using precision and recall measurement. Precision is the ratio of the number of relevant images captured to the number of retrieved images. The formula for calculating precision symbolized by $\mathrm{Pr}$ is as follows [9]:

$$
\operatorname{Pr}=\frac{\text { Total Relevan Retrieved Image }}{\text { Total Number of Retrieved Images }}
$$

Recall is the ratio of the number of images relevant to the overall number of relevant images in the database. The formula for recall symbolized by $R c$ counting is as follows [9].

$$
R c=\frac{\text { Number of Relevan Image that are Retrieved }}{\text { Total Number of Relevant Image in the database }}
$$

F-Measure is a value representing all system performance which is the average of precision and recall value. The formula of F-Measure can be seen in the following equation [9]:

$$
F-\text { measure }=2 \cdot \frac{P r \cdot R c}{P r+R c}
$$

\subsection{Human Perception Measure Parameters}

In addition to calculating the recall, precision, and F-Measure values of the SCD and DCD color descriptor performance will also be compared with the perceptual results of human vision. This study also involves humans directly. There were 10 respondents who were asked for their willingness to fill out a questionnaire consisting of 5 color categories. For each category there were 2 queries. The total query was 10 queries. The results of the respondents opinions will be matched with the simulation result of the system using SCD and DCD features. The simulation that best suits the respondents perception was considered best suited to the female muslim clothing object. To express a better image feature than any other feature of the system based on human eye perception, it is necessary to know and calculate the effectiveness level [10], [13]. Effectiveness is calculated by comparing the rank between the simulation system and human perceptions. The higher the evectiveness value, proves more similar to the human perception.

$$
\text { Effectiveness }=\frac{1}{1+\log \left(\frac{R}{R c}\right)} \frac{\sum_{i=1}^{R c} i}{\sum_{i=1}^{R c} i+\sum_{i=1}^{R c}|i-r i|}
$$

Where: $R=$ Total number relevant color $(R \geq R c)$

$R c=$ Number of relevant colors selected (corresponding human perception)

$i=$ relevant color sequence $(i=1,2$,

$r i=$ sequence similarity image in system with Human perception (rank

\section{Result and Analisys}

This section discusses the analisys of CBIR system for women's clothing for each category using SCD and DCD color features. Furthermore, it would be known between the two features which are best used on the object of female Muslim fashion. SCD and DCD simulation 
results will also be compared with the perception of the human eye consisting of 10 respondents, in order to know the results of the simulation closest to human perception. The simulated SCD and DCD color feature simulation results were 10 color sequences that have the closest vector distance. The result of system simulation was compared with human perception.

Figure 3. shows the result of SCD simulation category blue. Figure 4. shows the result of DCD simulation. Figure 5. and Figure 6. shows data perception respondens 4 . Based on the simulation result of system for SCD and DCD, all top-10 result are displayed according to the query category. It correspondens to the human perception that also selects all image top-10 from the blue category. But there are different sequence of images generated simulation SCD, DCD and human persepsion. To know the color features that fit the human perception, then compared the result of the simulation system with human perspective. Based on comparison of simulation system SCD with respondent 4 there are 3 same sequence, that is at top-1, top-4, and top-5. As for DCD, the comparison with respondent 4 is 2 of the same sequence that is at top-1, top-4. While simulation comparison SCD and respondent 10, is 1 of the same sequence on top. And DCD there are 2 same sequence, that is at top-1 and top-5. The effectiveness value obtained for the SCD feature is 0.4161 and for DCD is 0.4235 .

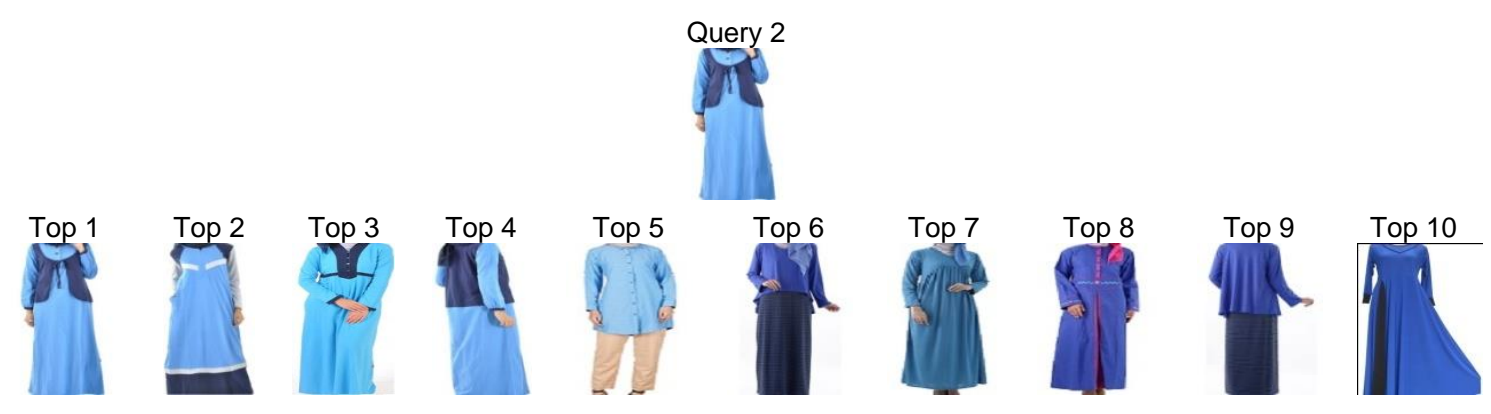

Figure 3. SCD simulation
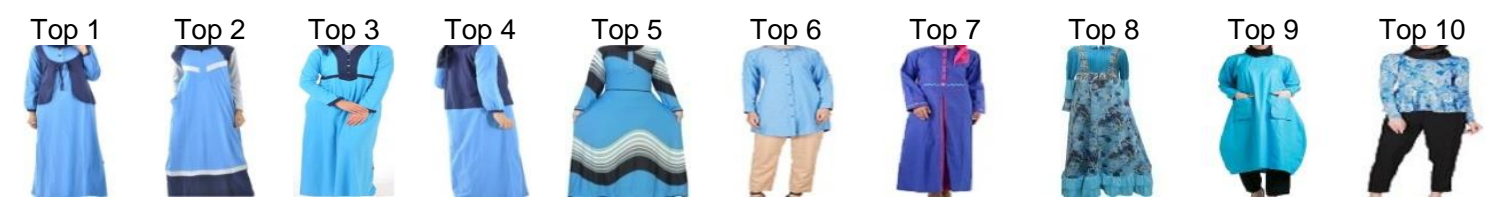

Figure 4. DCD simulation
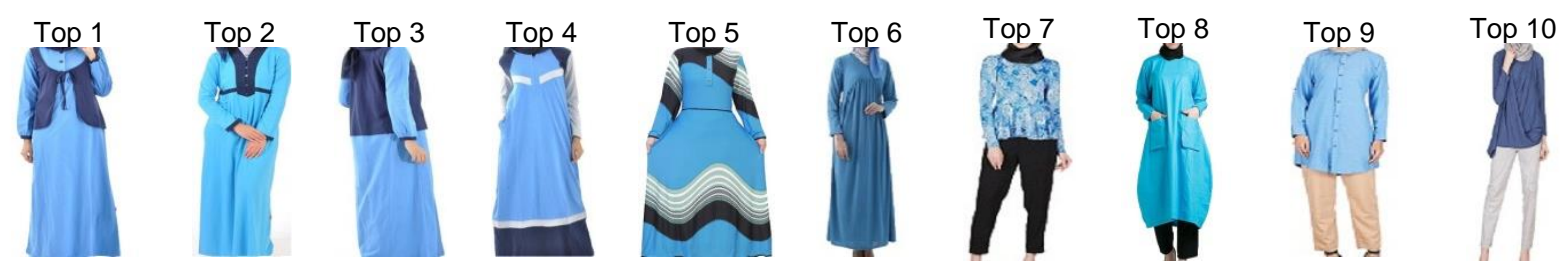

Figure 5. Perception of respondents 10
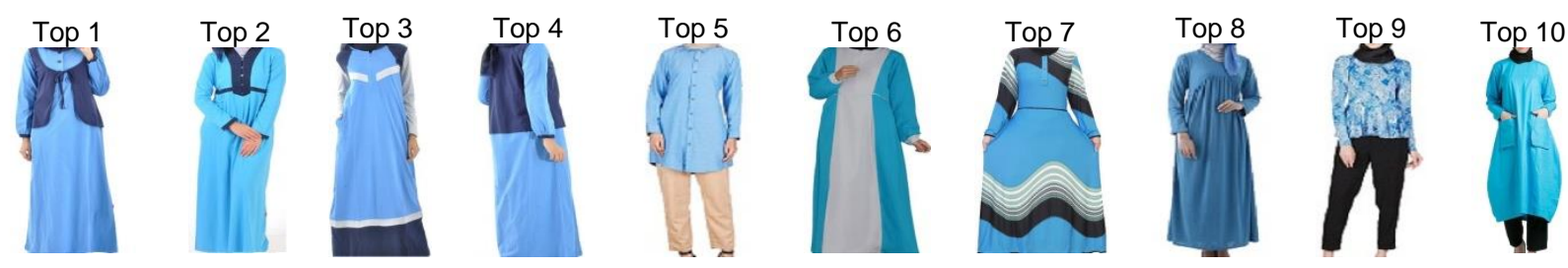

Figure 6. Perception of respondents 4

Bulletin of EEI Vol. 6, No. 2, June 2017 : 166-173 
Figure 7. and Figure 8. shows simulation result of system for SCD there were 3 images that did not come from red category that was on top-8, top-9, and top-10. It did not match the query image coming from the red category. As for DCD, all imagery results derived from the red category. Based on comparison of simulation system with human perception shown in Figure. 3 is to feature SCD with respondent 5 . There are 1 same sequence, that is at top-1. As for DCD, the comparison with respondent 5 is 1 of the same sequence on top. While simulation comparison SCD and respondent 9, is 1 of the same sequence on top. And DCD there are 2 same sequence, that is at top-1 and top-7. The effectiveness value obtained for the SCD feature is 0.389 and for DCD is 0.407 .

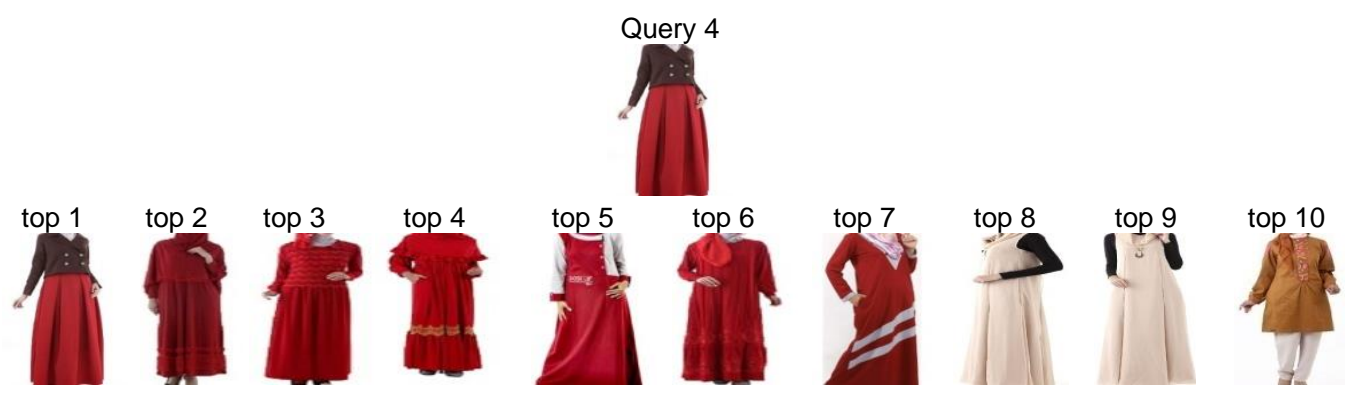

Figure 7. SCD simulation

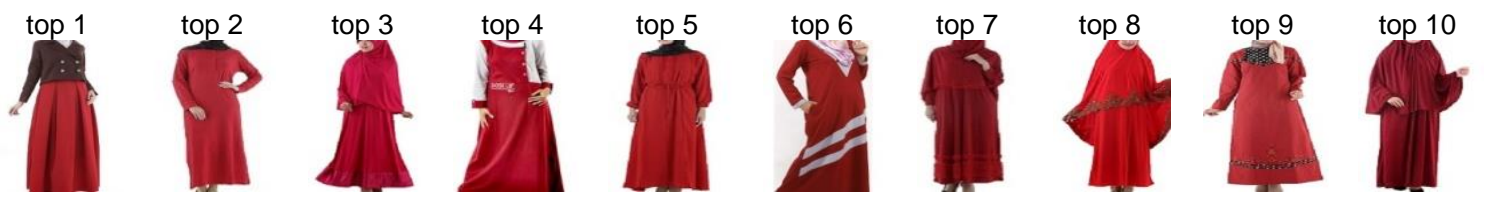

Figure 8. DCD simulation
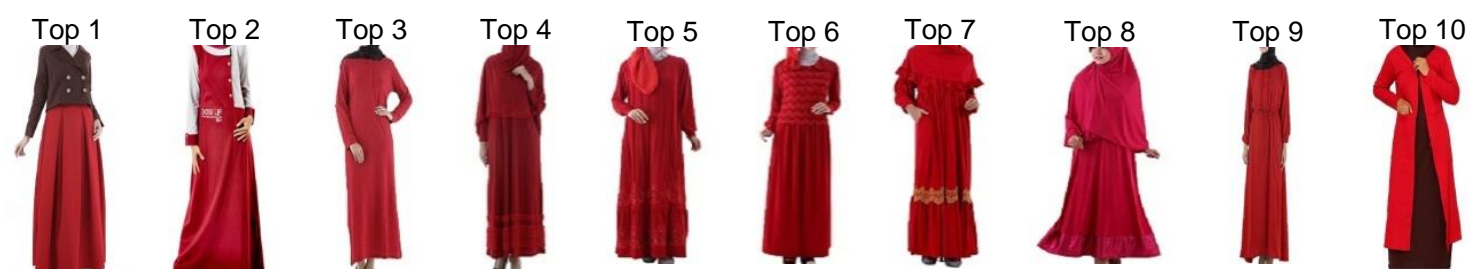

Figure 9. Perception of respondents 5

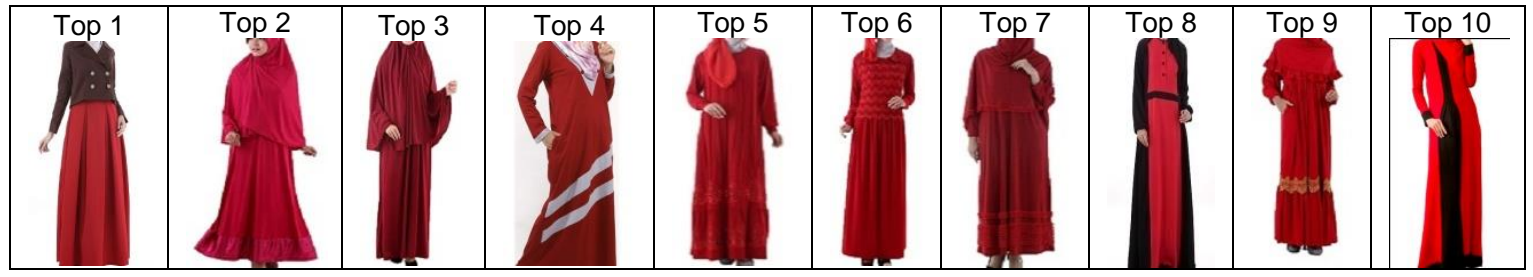

Figure 10. Perception of respondents 9 


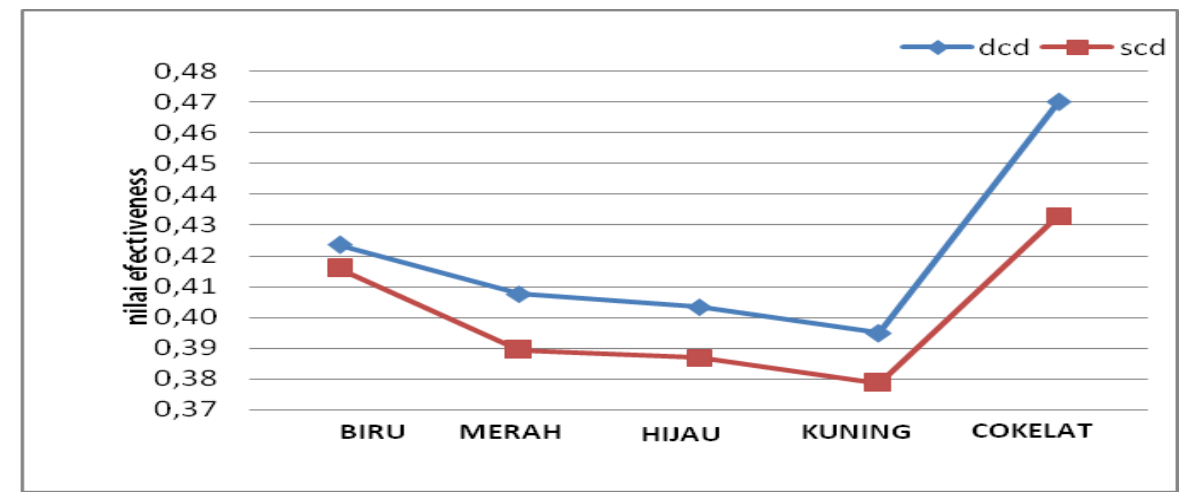

Figure 10. Effectiveness color feature SCD and DCD

Comparison of simulation results using SCD and DCD features will be adjusted to the perception of the human eye. The comparison result will be measured by effectiveness [10]. Respondents consisted of 10 people. Each respondent was given 10 queries and 150 dataset of female Muslim fashion. Respondents were asked to select the 10 most closely colored results of the query according to the respondents' perceptions of the eyes. From each respondent opinion will search the similarity sequence (rank) image with the results of CBIR system simulation on the order of top-10. From the data of 10 respondents, we will look for the sequence which has the same rank with the simulation result of the system using SCD color feature and DCD feature. The average value of the results obtained would be acquired for each category to determine the best color features used in the object of female Muslim fashion. The highest average value shows that the color features were most appropriate to the perception of the eyes vision of humans in choosing Muslim fashion based on color. From the results obtained shows that the comparison of human perception with DCD color features have higher efectiveness value than comparison by using SCD. This happen because the perception of the Muslim fashion order chosen by humans is more in line with the CBIR simulation results using the DCD color feature. From the CBIR system simulation results and based on the comparison of human perception, showing that the DCD color feature is more suitable for women muslem clothing. The reason is because in search of fabric, women generally wear the dominant color of an image by ignoring the small component of color contained in clothing.

\section{Conclusion}

The MPEG-7 color descriptors SCD and DCD were successfully applied for extracting color features on the CBIR system of female Muslim fashion. Used as many as 150 women Muslim clothing consisting of 5 color categories. SCD and DCD provide good results in the CBIR system of Muslim fashion, as it can display color results similar to queries. Based on the simulation system of the two color descriptor, DCD gives better result to apply to woman moslem fashion object. In almost all color categories, the F-Measure DCD value provides a higher value than the SCD. For example, for some categories in the red category, the FMeasure value obtained is 50\% for the DCD feature and $40 \%$ for SCD features. In blue category $60 \%$ for DCD and $50 \%$ for SCD. In the $48 \%$ chocolate category for DCD and $37 \%$ for SCD. SCD and DCD simulation results were also adjusted to direct human analysis consisting of 10 respondents. Based on this, the effectiveness value obtained for DCD was also higher than that of SCD. For blue category was obtained value of 0.435 for DCD and 0.416 for SCD. In red category 0.407 for DCD and 0.389 for SCD. In the Green category 0.403 for DCD and 0.387 for SCD. In the yellow category 0.394 for DCD and 0.378 for SCD. As for the chocolate category 0.478 for DCD and 0.432 for SCD. This happens because DCD displays only the most dominant colors only, so it is very much in line with human vision that generally sees the dominant color of an object and ignores the few color components. While on SCD, all color proportions contained in the query will search for its resemblance even if only small color components. In addition, Muslim fashion that usually consists of a combination of several colors also make the DCD more suitable features applied in the Muslim clothing search system. 


\section{References}

[1] MJ Quan. Contend Based Image Retrieval with HSV Color Space and Texture Features. IEEE International Conferencess on Web Information System and Mining. 2009; 2.

[2] R Jens, K Jun Heon, M Dean S. The MPEG-7 Color Descriptor. https://pdfs.semanticscholar.org.

[3] B Thomas. Scalable Color Graphics and Group of Frames/Group of Pictures. Summary and Details by Thomas Bucsics. August 2004.

[4] S Thomas. The MPEG-7 Visual Standar for Content Description-An Overview. IEEE Transaction on Circuits and System for Video Technology. June 2001; 11(6).

[5] S Hong, W Yueshu, C Wencheng. Image Retrieval Based on MPEG-7 Dominant Color Descriptor. IEEE Internationsl Conference for Young Computer Scientists. 2008

[6] R Errick, M Cahyatai. Algoritma Transformasi Ruang dan Warna. Juli 2010

[7] L Vedran, S Haris. A Compact Color Descriptor for Image Retrieval. IEEE Information, Comunication, and Automation Technologies (ICAT). 2013

[8] F Windana, M Marosa, PB Santoso. Implementasi Kombinasi Feature Extraction untuk Content Based Image Retrieval. EECIS International Conference on Electric Power, Electronic, Communication, Control and Informatic System. 2014; 8(2).

[9] S Nikil Vasudev. Evaluating Image Retrieval. Master of Science in Electrical and Computer Enginering, Universitas of Arizona. 2005

[10] Sarifuddin M, R Missaori. A New Perceptually Uniform Color Space with Associated Color Similarity Measure for Content Based Image and Video Retrieval. Proceeding of Multimedia Information Retrieval Workshop, $28^{\text {th }}$ annual ACM SIGIR Conference. 2005:1-8.

[11] D Yustina, M Khairul, A Fitri. Penerapan Deskriptor Warna Dominan untuk Temu Kembali Citra Busana Pada Peranti Bergerak. Jurnal Rekayasa Elektrika. 2016; 12(3):104-110.

[12] C Falaoutsos, W Equitz, M Flickner, W Niblack, D Petkovic, R Barber. Efficient and Effective Querying Mixture. Tech.Rep., Dep, Cs. Universitas de Sherbrooke,2003.

[13] R Missaoui, M Sarifuddin, J Vaillancourt. Similarity Measure for Efficient Content Based Image Retrieval. IEE Proc.-Vis.Image Signal Process. December 2005; 152(6). 